\title{
A Mean- maximum Deviation Portfolio Optimization Model
}

\author{
Wu Jinwen \\ School of Economic and Management, South China Normal University \\ Guangzhou 510006, China
}

Tel: 86-138-0299-1363Ｅ-mail: wujinwen@139.com

\begin{abstract}
The essay makes a thorough and systematic study about a mean- maximum deviation portfolio optimization model. First, we make a careful analysis about the problem and build a model about this kind of problem. The essay gives two kind of different and characteristic solutions-linear programming solution and critical line solution.
\end{abstract}

Key words: Expected yield rate, Risk, Critical line, Maximum risk-measurement

\section{Introduction}

In the path-breaking work on Portfolio Selection, Markowitz (1952) developed the concept of an efficient portfolio in terms of the expected return and standard deviation of return. Modern Portfolio Theory (MPT) has become one of the most important bases in modern capital market, and been applied widely in the practice of investment. In the absence of specific knowledge of investor's preference, however, it cannot be determined which of any two efficient portfolios is better. Baumel (1963) replaced the $(E, \sigma)$ criteria with the $(E, E-k \sigma)$ criteria, where $k$ stands for the investor's attitude toward risk. Baumol demonstrated that his $(E, E-k \sigma)$ criteria yield a smaller efficient set, which is a subset of the Markowitz efficient set, and therefore reduces the range of alternatives from which the investor has to select his portfolio. Recently, Li (2000) studied the optimal portfolio selection with transaction costs. Hiroshi Konno discussed the mean-absolute deviation portfolio optimization model and its applications.

In this paper we discuss the mean- maximum deviation portfolio optimization model. More precisely, we seek to define portfolio that produces maximal yield and at the same time satisfies constraints on minimum risk. Our aim is to develop a theory similar to Markowitz theory for optimal mean-variance portfolios and provide algorithmic tools for computing such portfolios. Our emphasis here is on algorithms because, unlike optimal mean-variance portfolios, the meanmaximum deviation portfolio optimization model generally defy analysis with simple analytical tools.

Let's consider such a problem; assume a company selects kinds of securities $S_{i}(i=1,2, \Lambda, n)$ to invest. The expected yield rate of $S_{i}$ is $R_{i}$, and the risk of $S_{i}$ is $q_{i}$. The company defines that the total risk of portfolio is measured by the maximum risk of $S_{i}$. Assume the interest rate is $r_{0}$ at the same period and without any risk. How to define the investing weight of the a kinds of securities to make the expected yield rate of the portfolio to possible maximum, and the total risk to possible minimum.

Denote the risk of portfolio is $q_{c}$, the expected yield rate of the portfolio is $R_{c} \cdot x_{i}$ is the investing weight of bought assets $S_{i}(i=1,2, \Lambda, n), x_{0}$ is the investing weight of bank savings. Then

$$
x_{0}+x_{1}+x_{2}+\Lambda+x_{n}=1 \quad x_{i} \geq 0 \quad(i=0,1,2, \Lambda, n)
$$

According to the requirements of the problem, we can get the following model:

$$
\begin{cases}\min q_{c}=\max _{1 \leq i \leq n}\left\{x_{i} q_{i}\right\} & \\ & R_{c}=x_{0} r+x_{1} R_{1}+x_{2} R_{2}+\Lambda+x_{n} R_{n} \\ \text { s.t. } & x_{0}+x_{1}+x_{2}+\Lambda+x_{n}=1 \\ & x_{i} \geq 0 \quad(i=0,1,2, \Lambda n)\end{cases}
$$

or 


$$
\begin{cases}\max & R_{c}=x_{0} r+x_{1} R_{1}+x_{2} R_{2}+\Lambda+x_{n} R_{n} \\ & q_{c}=\max _{1 \leq i \leq n}\left\{x_{i} q_{i}\right\} \\ \text { s.t. } & x_{0}+x_{1}+x_{2}+\Lambda+x_{n}=1 \\ & x_{i} \geq 0 \quad(i=0,1,2, \Lambda n)\end{cases}
$$

\section{The linear programming solution of the model}

Now let's consider the model (I) only.

(a) Denote

$$
X=\left\{x=\left(x_{0}, x_{1}, \Lambda, x_{n}\right) \in R^{n+1} \mid x_{0}+x_{1}+x_{2}+\Lambda+x_{n}=1, \quad x_{0} r_{0}+\sum_{i=1}^{n} x_{i} R_{i}=R_{c}, \quad x \geq 0\right\}
$$

Then we know that the feasible set $X$ is a closed bounded polyhedral convex set.

(b) $q_{c}=\max _{1 \leq i \leq n}\left\{x_{i} q_{i}\right\} \quad\left(q_{i} \in(0,1), \quad i=1,2, \Lambda, n\right)$

$q_{c}$ is the point supreme of $n$ linear functions in $X$. So it's a continuous bounded linear convex function. According to (a)(b), $\min _{x \in X} q_{c}$ must have the optimal solution.

(c) Solution: Denote

$$
\begin{gathered}
X_{i}=\left\{x=\left(x_{0}, x_{1}, \Lambda, x_{n}\right) \in X \mid x_{i} q_{i} \geq x_{1} q_{1}, \Lambda, x_{i} q_{i} \geq x_{i-1} q_{i-1}, x_{i} q_{i} \geq x_{i-1} q_{i-1}, \Lambda, x_{i} q_{i} \geq x_{n} q_{n}\right\} \\
i=1,2, \Lambda \quad, \quad n
\end{gathered}
$$

Then $q_{c}=x_{i} q_{i}$ in $X_{i}$.

Solve $n$ problems of linear programming $L P_{i}$ separately:

$$
\left\{\begin{array}{l}
\min x_{i} q_{i} \\
\text { s.t. } \quad x \in X_{i}
\end{array}\right.
$$

Apparently all these problems have optimal solutions. Suppose the optimal solution of $L P_{i}$ is $x^{i^{*}}$, the optimal value is

$$
q_{i}^{*}=x_{i}^{i *} q_{i} \quad i=1,2, \Lambda, n
$$

Here, $x_{i}^{i *}$ is the $i$ th component of $x^{i^{*}}$.

Denote $\min _{1 \leq i \leq n} q_{i}^{*}=q_{k}^{*}=x_{k}^{k *} q_{k}$, then $x^{k^{*}}$ is the optimal solution of $(I)$, and $q_{k}^{*}=x_{k}^{k *} q_{k}$ is the optimal value of $(I)$.

Using this method, under a group of given expected yield rate of the portfolio, we can get a relevant group of optimal weights to minimum its risk.

\section{The critical line solution of the model}

Now we consider the model $(I)$ and $(I I)$.

Suppose $0<q_{1}<q_{2}<\Lambda<q_{n}$, and $r_{0}<R_{1}<R_{2}<\Lambda<R_{n}$. For $x_{0}+x_{1}+x_{2}+\Lambda+x_{n}=1$, we have $x_{0}=1-x_{1}-x_{2}-\Lambda-x_{n}$. So the expected yield rate and risk of portfolio is respectively:

$$
\left\{\begin{array}{l}
R_{c}=x_{0} r_{0}+x_{1} R_{1}+\Lambda+x_{n} R_{n}=r_{0}+\sum_{i=1}^{n}\left(R_{i}-r_{0}\right) x_{i} \\
q_{c}=\max _{1 \leq i \leq n}\left\{x_{i} q_{i}\right\}
\end{array}\right.
$$

Similarly, we can define the concepts of iso-expected-yield-rate super-plane of portfolio, iso-risk super-curved-surface of portfolio, critical line, etc.

Difinition 1: In the weight space $\left(x_{1}, x_{2}, \Lambda, x_{n}\right)$, given the expected yield rate of portfolio $R_{c}$, the super-plane defined by the equation (1) is called an iso-expected-yield-rate super-plane of portfolio. All the portfolios on this 
super-plane have the same expected yield rate. With different $R_{c}$, we can get a family parallel iso-expected-yield super-plane such as $f_{1}$ or $f_{3}$ in figure 1 . With the increasing of $x_{1}$, the yield rate is presented by the iso-expected-yield-rate line increase steadily.

Difinition 2: In the weight space $\left(x_{1}, x_{2}, \Lambda, x_{n}\right)$, given the risk of portfolio $q_{c}$, the super-curved-surface defined by the equation (2) is called an iso-risk super-curved-surface of portfolio. All the portfolios on this super-curved-surface have the same risk. With different $q_{c}$, we can get a family parallel iso-risk super-curve such as $b_{1}$ or $b_{5}$ in figure 1 . With the line segment $\mathrm{OB}$ from $\mathrm{O}$ to $\mathrm{B}$, the risk presented by the iso-risk line increases steadily.

Denote a iso-expected-yield super-plane (such as $f_{3}$ in figure 1). Above the segment $\mathrm{OB}$, the risk on the so-expected-yield super-plane decrease from the top down to the point of intersection (point $\mathrm{M}$ ) of this plane and the segment OB. Below the segment $\mathrm{OB}$, the risk on this plane decreases from the right to the left, until to the point of intersection (point $\mathrm{M}$ ) of this plane and the segment OB. We call the point of intersection the first kind of tangency point of the so-expected-yield super-plane and the iso-risk super-curve; we call the locus of these points of intersection the first kind critical line. Its equation is

$$
x_{1} q_{1}=x_{2} q_{2}=x_{3} q_{3}=\Lambda=x_{n} q_{n} .
$$

In the weight space $\left(x_{1}, x_{2}, \Lambda, x_{n}\right)$, the first kind critical line intersects with the boundary of investment area at point $H_{1}$. For he same reason, we can define the second critical line, and its equation is

$$
\left\{\begin{array}{l}
x_{2} q_{2}=x_{3} q_{3}=\Lambda=x_{n} q_{n} \\
x_{1}+x_{2}+\Lambda+x_{n}=1
\end{array}\right.
$$

Furthermore, we can get the equation of the $k$ th critical line:

$$
\left\{\begin{array}{l}
x_{k} q_{k}=x_{k+1} q_{k+1}=\Lambda=x_{n} q_{n} \\
x_{1}=x_{2}=\Lambda=x_{k-2}=0 \\
x_{k-1}+x_{k}+\Lambda+x_{n}=1
\end{array}\right.
$$

When the given iso-expected-yield super-plane does not intersect the segment $\mathrm{OB}$ in $\triangle O A B$, the plane should intersect the line $\mathrm{BC}$ (such as $f_{6}$ ), which the decreasing of $x_{2}$, the risk on the plane decrease steadily and come to min at the point of intersection (point B) of the plane and the segment BC. We call this point the $n$th point of tangency of the iso-expected-yield super-plane and the iso-risk super curve, and call the locus of those points of tangency the $n$th critical line. Its equation is:

$$
\left\{\begin{array}{l}
x_{1}=x_{2}=\Lambda=x_{n-2}=0 \\
x_{n-1}+x_{n}=1
\end{array}\right.
$$

The first critical line, the second critical line, ..., the $n$th critical line are all called the critical line of portfolio. According to the definition, the critical line of portfolio is a continuous space broken line, we mark the broken point respectively as: $H_{1}, H_{2}, \Lambda, H_{n-1}$. According the definition of critical line, given any an expected yield rate, we can find the optimal weight of portfolio, which make the risk minimum on critical line. At the same time, given any a denoted risk of portfolio, we can find the optimal weight of portfolio that can make the expected yield rate maximum on critical line. So we need only to solve the critical line equation of portfolio, in order to find the solution of model $(I)$ or $(I I)$.

According to the definition of critical line, the risk, the expected yield rate and the weight at point $H_{k}(k=1,2, \Lambda, n-1)$ should be respectively:

$$
\begin{gathered}
q_{c}^{k}=\left(\sum_{i=k}^{n} \frac{1}{q_{i}}\right)^{-1} \\
R_{c}^{k}=\frac{q_{c}^{k}}{q_{k}} R_{k}+\frac{q_{c}^{k}}{q_{k+1}} R_{k+1}+\Lambda+\frac{q_{c}^{k}}{q_{n}} R_{n} \\
\left(x_{0}, x_{1}, \Lambda, x_{k}, \Lambda, x_{n}\right)=\left(0, \Lambda, 0, \frac{q_{c}^{k}}{q_{k}}, \frac{q_{c}^{k}}{q_{k+1}}, \Lambda, \frac{q_{c}^{k}}{q_{n}}\right)
\end{gathered}
$$

\section{An Example}


Suppose there are four securities: $S_{1}, S_{2}, S_{3}, S_{4}$, their expected yield rate and risks are:

$$
R_{1}=0.074, \quad R_{2}=0.281, \quad R_{3}=0.339, \quad R_{4}=0.434
$$

According to equations (3), (4), (5), we know:

At point $H_{1}, q_{c}^{1}=0.0869, R_{c}^{1}=0.2376,\left(x_{0}, x_{1}, \Lambda, x_{4}\right)=\left(\begin{array}{llll}0, & 0.3778, & 0.2602, & 0.2173,0.1448\end{array}\right)$.

At point $H_{2}, q_{c}^{2}=0.1397, R_{c}^{2}=0.337,\left(x_{0}, x_{1}, \Lambda, x_{4}\right)=\left(\begin{array}{llll}0,0.4183,0.3493,0.2328\end{array}\right)$.

At point $H_{3}, q_{c}^{3}=0.24, R_{c}^{3}=0.377,\left(x_{0}, x_{1}, \Lambda, x_{4}\right)=\left(\begin{array}{llll}0, & 0,0,0.6 & 0.4\end{array}\right)$

So the critical line should be:

The first critical line equation is: $0.23 x_{1}=0.334 x_{2}=0.4 x_{3}=0.6 x_{4}$.

The second critical line equation is: $\left\{\begin{array}{l}0.334 x_{2}=0.4 x_{3}=0.6 x_{4} \\ x_{1}+x_{2}+x_{3}+x_{4}=1\end{array}\right.$

The third critical line equation is: $\left\{\begin{array}{l}0.4 x_{3}=0.6 x_{4} \\ x_{2}+x_{3}+x_{4}=1 \\ x_{1}=0\end{array}\right.$

The forth critical line equation is: $\left\{\begin{array}{l}x_{1}=x_{2} \\ x_{3}+x_{4}=1\end{array}\right.$

With different $R_{c}$ or $q_{c}$, using critical line equation (1) or (2), we can find respectively their optimal weight (See Table 1 or 2). With different $R_{c}$, using the linear programming solution, we can find their optimal weight (Table 1).

\section{Conclusion}

Our essay gives two ways to solve a mean- maximum deviation portfolio optimization model. One is the linear programming solution. The other is the critical line solution. Both of the solution can find the optimal solution of the problem, and both the simulated numbers results are identical. The linear programming solution can only find the optimal weight of portfolio which make the risk to minimum while given the expected yield rate, but it is helpless to find the optimal weight of portfolio which make the expected yield rate to maximum while given the risk. And the linear programming solution can only find the solution under given data. However, the critical line solution can resolve both of these problems.

\section{Reference}

Harry. Markowitz, Portfolio Selection, Journal of Finance, March 1952, 77-91

Baumol W J, An Expected Gain-Confidence Limit Criterion for Portfolio Selection, Management Science, 1963, 10. 174-182.

Li Z F, Wang S Y and Deng X T, A Linear Programming Algorithm for Optimal Portfolio Selection with Transaction Costs, International Journal of Systems Science, 2000, 31(1). 107-117.

Hiroshi Konno and Annista Wijayanayake, Mean-Absolute Deviation Portfolio Optimization Model under Transaction Costs, Journal of the Operations Research Society of Japan, 1999, 42(4). 422-435.

Table 1. Given different $R_{c}$, the optimal weight of portfolio and its min-risk

\begin{tabular}{|l|l|l|l|l|l|l|l|l|l|l|}
\hline$R_{c}$ & 0.22 & 0.23755 & 0.26 & 0.28 & 0.30 & 0.32 & 0.33686 & 0.36 & 0.377 & 0.4 \\
\hline$x_{0}$ & 0.09359 & 0 & 0 & 0 & 0 & 0 & 0 & 0 & 0 & 0 \\
\hline$x_{1}$ & 0.34243 & 0.37779 & 0.29240 & 0.21632 & 0.14023 & 0.06414 & 0 & 0 & 0 & 0 \\
\hline$x_{2}$ & 0.23581 & 0.26816 & 0.29586 & 0.32767 & 0.35949 & 0.39130 & 0.41812 & 0.17708 & 0 & 0 \\
\hline$x_{3}$ & 0.19698 & 0.21723 & 0.24704 & 0.27361 & 0.30017 & 0.32674 & 0.34913 & 0.49375 & 0.6 & 0.35790 \\
\hline$x_{4}$ & 0.13127 & 0.14482 & 0.16470 & 0.18241 & 0.20011 & 0.21782 & 0.23275 & 0.32917 & 0.4 & 0.64211 \\
\hline$q_{c}$ & 0.07876 & 0.08689 & 0.09882 & 0.10944 & 0.12007 & 0.13069 & 0.13965 & 0.19750 & 0.24 & 0.38526 \\
\hline
\end{tabular}


Table 2. Given different $q_{c}$, the optimal weight of portfolio and its max-expected-yield-rate

\begin{tabular}{|l|l|l|l|l|l|l|l|l|l|l|}
\hline$q_{c}$ & 0.05 & 0.07 & 0.08689 & 0.10 & 0.12 & 0.13965 & 0.16 & 0.20 & 0.24 & 0.28 \\
\hline$x_{0}$ & 0.42458 & 0.19441 & 0 & 0 & 0 & 0 & 0 & 0 & 0 & 0 \\
\hline$x_{1}$ & 0.21739 & 0.30435 & 0.37779 & 0.28393 & 0.14072 & 0 & 0 & 0 & 0 & 0 \\
\hline$x_{2}$ & 0.14970 & 0.20958 & 0.26016 & 0.29940 & 0.35928 & 0.41812 & 0.33333 & 0.16667 & 0 & 0 \\
\hline$x_{3}$ & 0.125 & 0.175 & 0.21723 & 0.25 & 0.3 & 0.34913 & 0.4 & 0.5 & 0.6 & 0.53333 \\
\hline$x_{4}$ & 0.08333 & 0.11667 & 0.14482 & 0.16667 & 0.2 & 0.23275 & 0.26667 & 0.33333 & 0.4 & 0.46667 \\
\hline$R_{c}$ & 0.15792 & 0.20109 & 0.23755 & 0.26223 & 0.29988 & 0.33686 & 0.345 & 0.361 & 0.377 & 0.38333 \\
\hline
\end{tabular}



Figure 1. 